\title{
Stimulated scattering of electromagnetic waves carrying orbital angular momentum in quantum plasmas
}

P K Shukla, B Eliasson and Lennart Stenflo

\section{Linköping University Post Print}

N.B.: When citing this work, cite the original article.

Original Publication:

P K Shukla, B Eliasson and Lennart Stenflo, Stimulated scattering of electromagnetic waves carrying orbital angular momentum in quantum plasmas, 2012, Physical Review E. Statistical, Nonlinear, and Soft Matter Physics, (86), 1, 016403.

http://dx.doi.org/10.1103/PhysRevE.86.016403

Copyright: American Physical Society http://www.aps.org/

Postprint available at: Linköping University Electronic Press

http://urn.kb.se/resolve?urn=urn:nbn:se:liu:diva-79638 


\title{
Stimulated scattering of electromagnetic waves carrying orbital angular momentum in quantum plasmas
}

\author{
P. K. Shukla, ${ }^{1,2,{ }^{*}}$ B. Eliasson, ${ }^{1}$ and L. Stenflo ${ }^{3, \dagger}$ \\ ${ }^{1}$ International Centre for Advanced Studies in Physical Sciences \& Institute for Theoretical Physics, Faculty of Physics \& Astronomy, \\ Ruhr-University Bochum, D-44780 Bochum, Germany \\ ${ }^{2}$ Department of Mechanical and Aerospace Engineering \& Center for Energy Research, University of California San Diego, \\ La Jolla, California 92093, USA \\ ${ }^{3}$ Department of Physics, Linköping University, SE-58183, Linköping, Sweden
}

(Received 24 May 2012; published 12 July 2012)

\begin{abstract}
We investigate stimulated scattering instabilities of coherent circularly polarized electromagnetic (CPEM) waves carrying orbital angular momentum (OAM) in dense quantum plasmas with degenerate electrons and nondegenerate ions. For this purpose, we employ the coupled equations for the CPEM wave vector potential and the driven (by the ponderomotive force of the CPEM waves) equations for the electron and ion plasma oscillations. The electrons are significantly affected by the quantum forces (viz., the quantum statistical pressure, the quantum Bohm potential, as well as the electron exchange and electron correlations due to electron spin), which are included in the framework of the quantum hydrodynamical description of the electrons. Furthermore, our investigation of the stimulated Brillouin instability of coherent CPEM waves uses the generalized ion momentum equation that includes strong ion coupling effects. The nonlinear equations for the coupled CPEM and quantum plasma waves are then analyzed to obtain nonlinear dispersion relations which exhibit stimulated Raman, stimulated Brillouin, and modulational instabilities of CPEM waves carrying OAM. The present results are useful for understanding the origin of scattered light off low-frequency density fluctuations in high-energy density plasmas where quantum effects are eminent.
\end{abstract}

DOI: 10.1103/PhysRevE.86.016403

PACS number(s): 52.35.Fp, 52.35.Mw

\section{INTRODUCTION}

Recently, there has been growing interest in investigating collective nonlinear processes [1-4] in dense quantum plasmas, which are ubiquitous in a variety of physical environments (e.g., the cores of Jupiter and white dwarf stars [5-9], neutron and quark stars [7], warm dense matter [10]), in compressed plasmas produced by intense laser beams [11], in pulsed thermonuclear fusion devices, as well as in processing devices for modern high-technology (e.g., semiconductors [12], in thin films and nanometallic structures [13], etc.). In fact, due to superdensity plasmas in the crust of dense neutron stars and in the cores of white dwarf stars, one [14] encounters the formation of ionic crystals (fully ionized carbon, oxygen, iron, etc.) embedded into a sea of degenerate Fermi gas of electrons (here the electron Fermi temperature exceeds the plasma electron and ion temperatures). There have also been suggestions $[15,16]$ that strongly coupled quantum plasmas can be produced in laboratory devices by using laser cooling methods, so that the plasma electron temperature could be comparable with the electron Fermi temperature so that the quantum effects become significant at high plasma number densities. In quantum plasmas, degenerate electrons obey the Fermi-Dirac statistics.

Theoretical investigations [17-21] of nonlinear phenomena associated with both electrostatic and electromagnetic waves in quantum plasma fluids have been carried out previously. These nonlinear studies were based on the generalized

\footnotetext{
*profshukla@yahoo.de

†'lennart.stenflo@physics.umu.se
}

quantum hydrodynamical (GQHD) equations [22-25] for nonrelativistic degenerate electron fluids supplemented by Poisson's and Maxwell's equations. The generalized electron momentum equation in the GQHD model includes the quantum statistical pressure [26,27] and quantum forces due to electron tunneling through the quantum Bohm potential [22,24,25,28,29], spin magnetization of Bohr electrons [30], as well as the electron exchange and correlation effects [31,32] due to the electron spin [33]. Quantum mechanical effects are relevant for solid density plasmas, where the interelectron distance is of the order of the atomic dimensions. Here overlapping of electron wave functions occurs due to the Heisenberg uncertainty and Pauli's exclusion principles [27]. Accordingly, one encounters novel nonlinear high-frequency (hf) dispersive wave phenomena [2] at nanoscales. Furthermore, in quantum plasmas, the electron and ion coupling parameters are $\Gamma_{e}=$ $e^{2} / a_{e} k_{B} T_{F}$ and $\Gamma_{i}=Z_{i}^{2} e^{2} / a_{i} k_{B} T_{i}$, respectively, where $e$ is the magnitude of the electron charge, $a_{e} \sim a_{i}=\left(3 / 4 \pi n_{0}\right)^{1 / 3}$ the Wigner-Seitz radius, $n_{0}$ the unperturbed electron number density, $k_{B}$ the Boltzmann constant, $Z_{i}$ the ion charge state, $T_{i}$ the ion temperature, $T_{F}=\left(\hbar^{2} / 2 m_{0} k_{B}\right)\left(3 \pi^{2} n_{0}\right)^{2 / 3}$ the electron Fermi temperature in the nonrelativistic and zero-temperature limits, and $m_{0}$ the electron rest mass. It turns out that $\Gamma_{i} / \Gamma_{e}=Z_{i}^{2} T_{F} / T_{i} \gg 1$, since in quantum plasmas we usually have $T_{F}>T_{i}$. Recently, Glenzer et al. [34] have reported observations of enhanced electron plasma waves (plasmons) in solid density plasmas. Their measurements involved collective $\mathrm{x}$-ray scattering techniques that are capable of measuring the high-frequency plasma wave spectra, revealing a signature of quantum effects associated with the quantum statistical electron pressure and the quantum recoil of electrons, at electron number densities $\sim(1.5-4.5) \times 10^{23} \mathrm{~cm}^{-3}$ and at 
electron temperatures below $25 \mathrm{eV}$ (typically the electron temperature $T_{e}$ was $12 \mathrm{eV}$ in the experiments). The plasmon spectrum provides a sensitive measure of the electron number densities.

Large amplitude high-frequency electromagnetic (EM) waves are used for heating inertially confined fusion plasmas [35], as well as for diagnostic purposes [34] in solid density plasmas that are created by intense laser and charged particle beams. The hf EM pulses also appear as localized bursts of $\mathrm{x}$ rays and $\gamma$ rays from compact astrophysical objects. Furthermore, the generation of coherent hf EM waves is of great importance in the context of free-electron lasers involving EM wigglers [36,37]. Therefore, studies of nonlinear phenomena (e.g., parametric instabilities [17] and hf EM wave localizations [19]) associated with large amplitude hf EM waves in dense quantum plasmas are of practical interest.

In this paper, we present an investigation of stimulated scattering instabilities of coherent circularly polarized electromagnetic (CPEM) waves carrying orbital angular momentum (OAM) in an unmagnetized dense quantum plasma composed of nonrelativistic degenerate electron fluids and mildly coupled nondegenerate ion fluids. It should be noted that a recent work [38] has proposed a scheme to generate intense coherent light that carries OAM at the fundamental wavelength of an $\mathrm{X}$-ray free-electron laser (FEL). Our results reveal that quantum forces acting on degenerate electrons in quantum plasmas greatly modify the frequency spectra of both the electron and ion plasma oscillations that are driven at nonthermal levels by the CPEM wave pressure. Accordingly, the growth rates of stimulated Raman, stimulated Brillouin, and modulational instabilities are significantly affected when a large amplitude CPEM pump wave carrying OAM is present in an unmagnetized quantum plasma.

\section{FORMULATION}

The nonlinear interactions between the CPEM wave and the background dense quantum plasma are governed by the EM wave equation [17,39]

$$
\left(\frac{\partial^{2}}{\partial t^{2}}-c^{2} \nabla^{2}+\omega_{p}^{2}\right) \mathbf{A}+\omega_{p}^{2} N \mathbf{A}=0
$$

which is derived from the Maxwell equation and the electron equation of motion with the electromagnetic fields $\mathbf{E}=-c^{-1} \partial \mathbf{A} / \partial t$ and $\mathbf{B}=\nabla \times \mathbf{A}$, with the Coulomb gauge $\boldsymbol{\nabla} \cdot \mathbf{A}=0$. Here $\mathbf{A}$ is the vector potential, $c$ the speed of light in vacuum, $\omega_{p}=\left(4 \pi n_{0} e^{2} / m_{0}\right)^{1 / 2}$ the electron plasma frequency, $N=n_{e 1} / n_{0} \ll 1$, and $n_{e 1}$ the electron number density perturbation associated with low-frequency electrostatic plasma oscillations (EPOs) that are reinforced by the ponderomotive force of the CPEM waves. In the absence of nonlinear couplings between the latter and the EPOs, the paraxial EM wave solution $\mathbf{A}(r, z) \exp (-i \omega t+i k z)$, where $\omega=\left(k^{2} c^{2}+\omega_{p}^{2}\right)^{1 / 2}$ and $k$ are the frequency and the wave number, respectively, of Eq. (1) is [40,41]

$$
\mathbf{A}(r, z)=\mathbf{A} F_{p, l}(r, z) \exp (i l \varphi) .
$$

Here we have denoted

$F_{p, l}(r, z)=\frac{1}{2 \sqrt{\pi}}\left[\frac{(l+p) !}{p !}\right]^{1 / 2} X^{|l|} L_{p}^{|l|}(X) \exp (-X / 2)$,

where $X=r^{2} / w^{2}(z), w(z)$ is the beam waist, and the associated Laguerre polynomials $L_{p}^{|l|}(X)$ are defined by the Rodriguez formula $L_{p}^{|l|}(X)=\left(X^{l} p !\right)^{-1} \exp (X) d^{p}\left[X^{(l+p)}\right.$ $\exp (-X)] / d X^{p}, p$ and $l$ are the radial and angular mode numbers of the photon orbital angular momentum states, respectively, $\varphi$ is the azimuthal angle, and $r=\left(x^{2}+y^{2}\right)^{1 / 2}$ is the radial of the cylindrical coordinates $(r, \varphi, z)$, so that $\nabla^{2}=$ $\nabla_{\perp}^{2}+\partial_{z}^{2}$, where $\nabla_{\perp}^{2}=(1 / r)(\partial / \partial r)(r \partial / \partial r)+\left(1 / r^{2}\right) \partial^{2} / \partial \varphi^{2}$. The Laguerre-Gauss (LG) solutions (2) describe CPEM waves with a finite OAM.

The dynamics of low-frequency (in comparison with the CPEM wave frequency $\omega$ ) plasma oscillations involving degenerate electron and nondegenerate ion fluids is governed by a set of equations composed of the electron continuity equation

$$
\frac{\partial n_{e}}{\partial t}+\nabla \cdot\left(n_{e} \mathbf{u}_{e}\right)=0
$$

the electron momentum equation $[13,32]$

$m_{0} n_{e} \frac{d \mathbf{u}_{e}}{d t}-e n_{e} \nabla\left(\phi-\phi_{p}\right)+\nabla P_{e}-n_{e} \nabla V_{x c}-n_{e} \nabla V_{B}=0$,

and Poisson's equation

$$
\nabla^{2} \phi=4 \pi \rho,
$$

together with the ion continuity equation and the generalized viscoelastic ion momentum equation [2]. Here $n_{e}\left(n_{i}\right)$ is the electron number density, $\mathbf{u}_{e}$ the electron fluid velocity, $d / d t=\partial / \partial t+\mathbf{u}_{e} \cdot \nabla, \rho=e\left(n_{e}-n_{i}\right), \phi$ the electric potential, and $\phi_{p}=e|\mathbf{A}|^{2} / m_{0} c^{2}$ the ponderomotive potential of the CPEM waves. The light ponderomotive force [35,39] $-e \nabla \phi_{p}$ comes from the averaging (over the light wave period) of the advection and nonlinear Lorentz force involving the electron quiver velocity and the laser wave magnetic field. We have denoted the quantum statistical electron pressure [27] $P_{e}=\left(n_{0} m_{0} V_{*}^{2} / 5\right)\left(n_{e} / n_{0}\right)^{5 / 3}$, where $V_{*}=\hbar\left(3 \pi^{2}\right)^{1 / 3} / m_{0} r_{0}$ is the electron Fermi speed and $r_{0}=n_{0}^{-1 / 3}$ represents the WignerSeitz radius, and the sum of the electron exchange and electron correlation potential is $[31,32] V_{\mathrm{xc}}=-0.985 e^{2} n_{e}^{1 / 3}[1+$ $\left.\left(0.034 / a_{B} n_{e}^{1 / 3}\right) \ln \left(1+18.37 a_{B} n_{e}^{1 / 3}\right)\right]$, where $a_{B}=\hbar^{2} / m_{0} e^{2}$ represents the Bohr radius. The quantum Bohm potential is $[2,22] V_{B}=\left(\hbar^{2} / 2 m_{0}\right)\left(1 / \sqrt{n_{e}}\right) \nabla^{2} \sqrt{n_{e}}$. We have thus retained the desired quantum forces that act on degenerate electrons in a nonrelativistic quantum plasma. Equation (5) is valid $[2-4,23]$ if the plasmonic energy density $\hbar \omega_{\mathrm{pe}}$ is smaller than or comparable to the electron Fermi energy $k_{B} T_{F}$, and the electron-ion collision relaxation time is larger than the electron plasma period. 
The ion number density perturbation $n_{i 1}$ is obtained from the ion continuity equation

$$
\frac{\partial n_{i}}{\partial t}+\nabla \cdot\left(n_{i} \mathbf{u}_{i}\right)=0
$$

where the ion fluid velocity $\mathbf{u}_{i}$ is determined from the generalized viscoelastic ion momentum equation [2]

$$
\begin{gathered}
\left(1+\tau_{m} \frac{D}{D t}\right)\left[m_{i} n_{i} \frac{D \mathbf{u}_{i}}{D t}+\nabla P_{i}+Z_{i} e n_{i} \nabla \phi\right] \\
-\eta \nabla \cdot \nabla \mathbf{u}_{i}-\left(\xi+\frac{\eta}{3}\right) \nabla\left(\nabla \cdot \mathbf{u}_{i}\right)=0,
\end{gathered}
$$

where $D / D t=\partial / \partial t+\mathbf{u}_{i} \cdot \nabla, \quad \tau_{m}$ is the viscoelastic relaxation time for ion correlations, $P_{i}=\mu_{i} k_{B} T_{i} n_{i}$ the ion thermal pressure involving strong ion coupling effects, $m_{i}$ the ion mass, $\mu_{i}\left[=\left(1 / k_{B} T_{i}\right)\left(\partial P_{i} / \partial n_{i}\right)_{k_{B} T_{i}}\right] \equiv 1+U\left(\Gamma_{i}\right) / 3+$ $\left(\Gamma_{i} / 9\right) \partial U\left(\Gamma_{i}\right) / \partial \Gamma_{i}$ the isothermal compressibility factor for nondegenerate ion fluids [42], and the function $U\left(\Gamma_{i}\right)$ a measure of the excess internal energy of the system, which is related to the correlation energy $E_{c}$ by $U\left(\Gamma_{i}\right)=E_{c} / n_{i} k_{B} T_{i}$ $\left(\equiv \Gamma_{i}\left(0.9+1.5 r_{i}^{2} / a_{i}^{2}\right)\right.$, where $r_{i}$ is the ion core radius which depends on the degree of ion stripping [43]). For a onecomponent plasma model, one usually adopts $[44,45] U\left(\gamma_{i}\right) \approx$ $-0.9 \Gamma_{i}$ for $\Gamma_{i} \gg 1$. Furthermore, the coefficients of the shear and bulk ion fluid viscosities are denoted by $\eta$ and $\xi$, respectively. Thus, Eq. (8) is similar to that used by Frenkel [46] and Ichimaru and co-workers [44,45] in the context of ordinary fluids and one-component strongly coupled plasmas, respectively. Kaw and Sen [47] adopted a generalized viscoelastic dust momentum equation for studying the properties of dust acoustic waves [48] in multicomponent dusty plasmas with highly correlated coupled charged dust grains. Furthermore, we note that the low-frequency ponderomotive force (i.e., the gradient of the ion ponderomotive potential) of the high-frequency CPEM waves acting on the ion fluid is smaller by a factor $m_{0} / m_{i}$ as compared to $-\nabla \phi_{p}$, and therefore it has been neglected in Eq. (8).

Let us now derive the governing equations for the electron and ion plasma oscillations in the presence of the ponderomotive force of the CPEM wave in a quantum plasma. First, we consider the driven electron plasma oscillations on the time scale of the electron plasma period, so that the ions do not have time to respond and the ion density perturbation is zero. Letting $n_{e}=n_{0}+n_{e 1}$, where $n_{e 1} \ll n_{0}$, we linearize Eqs. (4)-(6) and combine the resultant equations to obtain the electron plasma wave equation

$$
\left(\frac{\partial^{2}}{\partial t^{2}}+\omega_{p}^{2}-U_{*}^{2} \nabla^{2}+\frac{\hbar^{2}}{4 m_{0}^{2}} \nabla^{4}\right) N=\frac{e^{2}}{2 m_{0}^{2} c^{2}} \nabla^{2}|\mathbf{A}|^{2},
$$

where we have denoted $U_{*}=\left(V_{*}^{2} / 3+C_{\mathrm{xc}}^{2}\right)^{1 / 2}$, with $C_{\mathrm{xc}}=$ $\left(0.328 e^{2} / m_{0} r_{0}\right)^{1 / 2}\left[1+0.62 /\left(1+18.36 a_{B} n_{0}^{1 / 3}\right)\right]^{1 / 2}$.

Second, we consider driven ion oscillations by supposing that $\left|\partial^{2} N / \partial t^{2}\right| \ll\left|U_{*}^{2} \nabla^{2} N-\left(\hbar^{2} / 4 m_{0}\right) \nabla^{4} N\right|$. Here, one can neglect the right-hand side in Eq. (5), and use the resultant equation to eliminate the electric field $-\nabla \phi$ from Eq. (8) to obtain, after linearization of the resultant equation under the quasineutral approximation $n_{e 1}=n_{i 1}$, the driven ion oscillation equation

$$
\begin{gathered}
\left(1+\tau_{m} \frac{\partial}{\partial t}\right)\left(\frac{\partial^{2}}{\partial t^{2}}-C_{s}^{2} \nabla^{2}+\frac{\hbar^{2}}{4 m_{0} m_{i}} \nabla^{4}\right) N \\
-\frac{\eta}{m_{i} n_{0}} \nabla \cdot \nabla \frac{\partial N}{\partial t}-\frac{\left(\xi+\frac{\eta}{3}\right)}{m_{i} n_{0}} \nabla^{2} \frac{\partial N}{\partial t} \\
=\left(1+\tau_{m} \frac{\partial}{\partial t}\right) \frac{Z_{i}^{2} e^{2}}{2 m_{0} m_{i} c^{2}} \nabla^{2}|\mathbf{A}|^{2},
\end{gathered}
$$

where we have used Eq. (7) to eliminate $\nabla \cdot \mathbf{u}_{i}$ and introduced $C_{s}^{2}=\left(V_{T i}^{2}+U_{*}^{2}\right)^{1 / 2}$, with $V_{T i}=\left(\mu_{i} k_{B} T_{i} / m_{i}\right)^{1 / 2}$.

Equations (1), (9), and (10) are the desired equations for studying the generation of wakefields [49] and nonlinear effects (viz., parametric instabilities [35] and localization of light pulses [39]) associated with LG CPEM beams in quantum plasmas at nanoscales.

\section{NONLINEAR DISPERSION RELATIONS AND THEIR ANALYSES}

In the following, we present an investigation of stimulated Raman, stimulated Brillouin, and modulational instabilities [50] of LG CPEM waves. Accordingly, we decompose the vector potential as

$$
\begin{aligned}
\mathbf{A}= & \mathbf{A}_{0+} \exp \left(-i \omega_{0} t+i \mathbf{k}_{0} \cdot \mathbf{r}\right)+\mathbf{A}_{0-} \exp \left(i \omega_{0} t-i \mathbf{k}_{0} \cdot \mathbf{r}\right) \\
& +\sum_{+,-} \mathbf{A}_{ \pm} \exp \left(-i \omega_{ \pm} t+i \mathbf{k}_{ \pm} \cdot \mathbf{r}\right)
\end{aligned}
$$

where the subscripts 0 and \pm denote the CPEM pump and CPEM sidebands, respectively, and $\omega_{ \pm}=\Omega \pm \omega_{0}$ and $\mathbf{k}_{ \pm}=$ $\mathbf{K} \pm \mathbf{k}_{0}$ are the frequency and wave vectors of the CPEM sidebands that are created by the beating of the pump $\left(\omega_{0}, \mathbf{k}_{0}\right)$ and electrostatic oscillations $(\Omega, \mathbf{K})$.

Inserting (11) into (1), (9), and (10), and supposing that $N$ is proportional to $\exp (-i \Omega t+i \mathbf{K} \cdot \mathbf{r})$, we Fourier decompose the resultant equations to obtain the nonlinear dispersion relations

$$
S_{R}=\frac{\omega_{p}^{2} e^{2} K^{2}}{2 m_{0}^{2} c^{2}} \sum_{+,-} \frac{\left|\mathbf{A}_{0} F_{p, l}\right|^{2}}{D_{ \pm}}
$$

and

$$
S_{B}=\frac{\omega_{p}^{2} Z_{i}^{2} e^{2} K^{2}}{2 m_{0} m_{i} c^{2}} \sum_{+,-} \frac{\left|\mathbf{A}_{0} F_{p, l}\right|^{2}}{D_{ \pm}} .
$$

Here we have denoted $S_{R}=\Omega^{2}-\omega_{p}^{2}-K^{2} U_{*}^{2}-\hbar^{2} K^{4} / 4 m_{0}^{2}$ and $S_{B}=\Omega^{2}-K^{2} C_{s}^{2}-\hbar^{2} K^{4} / 4 m_{0} m_{i}+i \Omega(\xi+4 \eta / 3) K^{2} /$ $m_{i} n_{0}\left(1-i \Omega \tau_{m}\right)$, and $D_{ \pm}=\omega_{ \pm}^{2}-k_{ \pm}^{2} c^{2}-\omega_{p}^{2} \approx \pm 2 \omega_{0}(\Omega-$ $\mathbf{K} \cdot \mathbf{V}_{g} \mp \delta$ ), where $\mathbf{V}_{g}=c^{2} \mathbf{k}_{0} / \omega_{0}$ is the group velocity of the CPEM pump, $\omega_{0}=\left(\omega_{p}^{2}+k_{0}^{2} c^{2}\right)^{1 / 2}$ the pump frequency, and $\delta=K^{2} c^{2} / 2 \omega_{0}$ the small frequency shift arising from the nonlinear interaction between the CPEM pump and the electrostatic plasma oscillations in a quantum plasma. In the absence of the pump, we have from (12) and (13) $S_{R}=0$ and $S_{B}=0$, which give the frequencies of the electron and ion plasma oscillations in quantum plasmas. We have

$$
\Omega(K)=\left(\omega_{p}^{2}+K^{2} U_{*}^{2}+\frac{\hbar^{2} K^{4}}{4 m_{0}^{2}}\right)^{1 / 2} \equiv \Omega_{L}
$$


for the electron plasma oscillations, and

$$
\Omega^{2}+i \Omega \frac{\Omega_{v}}{\left(1-i \Omega \tau_{m}\right)}-K^{2} C_{s}^{2}-\frac{\hbar^{2} K^{4}}{4 m_{0} m_{i}}=0
$$

for the ion plasma oscillations. Here we have denoted $\Omega_{v}=$ $(\xi+4 \eta / 3) K^{2} / m_{i} n_{0}$. In the hydrodynamic limit, viz., $\Omega \tau_{m} \ll$ 1 , we have viscous damping of the quantum ion mode. The real and imaginary parts of the frequencies $\left(\Omega=\Omega_{r}+i \Omega_{i}\right)$ are, respectively,

$$
\Omega_{r}(K)=\left[K^{2} C_{s}^{2}+\frac{\hbar^{2} K^{4}}{4 m_{0} m_{i}}-\Omega_{i}^{2}\right]^{1 / 2}
$$

and

$$
\Omega_{i}=-\frac{\Omega_{v}}{2}
$$

Furthermore, in the kinetic regime characterized by $\omega \tau_{m} \gg 1$, we have from (15)

$$
\Omega(K)=\left(\frac{\Omega_{v}}{\tau_{m}}+K^{2} C_{s}^{2}+\frac{\hbar^{2} K^{4}}{4 m_{0} m_{i}}\right)^{1 / 2} \equiv \Omega_{I}(K) .
$$

Generally, $\tau_{m}=\tau_{0} Y_{G}(K), \quad$ where $\quad \tau_{0}=1 / \Omega_{v}\left[1-\mu_{i}+\right.$ $\left.4 U\left(\Gamma_{i}\right) / 15\right]$ and $Y_{G}(K)=\exp \left(-K / K_{G}\right)$ for a Gaussian distribution, and $Y_{G}(k)=\left(1+K^{2} / K_{L}^{2}\right)^{-1}$ for a Lorentzian distribution. Here $K_{G}$ and $K_{L}$ are the scale factors [45].

We now present a summary of formulas for the growth rates of stimulated Raman (SR) and stimulated Brillouin (SB) scattering instabilities, as well as of modulational instabilities of a constant amplitude pump that is scattered off a quantum electron plasma wave, a quantum ion mode, and a spectrum of nonresonant electron and ion density perturbations. For threewave decay interactions, one assumes that $D_{-}=0$ and $D_{+} \neq$ 0 . Thus, one ignores $D_{+}$from Eqs. (12) and (13). Letting $\Omega=$ $\mathbf{K} \cdot \mathbf{V}_{g}-\delta+i \gamma_{R, B}$, and $\Omega=\Omega_{L}+i \gamma_{R}$ and $\Omega=\Omega_{r}\left(\Omega_{I}\right)+$ $i \gamma_{B}$ in the resultant equations, we obtain the growth rates for Raman and Brillouin backscattering $\left(|\mathbf{K}|=2 k_{0}\right)$ instabilities, respectively,

$$
\gamma_{R}=\frac{\omega_{p} k_{0} e\left|\mathbf{A}_{0} F_{p, l}\right|}{\sqrt{2 \omega_{0} \Omega_{R}} m_{0} c}
$$

and

$$
\gamma_{B}=\frac{\omega_{p} k_{0} Z_{i} e\left|\mathbf{A}_{0} F_{p, l}\right|}{\sqrt{2 \omega_{0} \Omega_{B} m_{0} m_{i}} c},
$$

where $\Omega_{R}=\Omega_{L}\left(K=2 k_{0}\right), \quad \Omega_{B}=\Omega_{r, I}\left(K=2 k_{0}\right)$, and $\left|2 \mathbf{k}_{\mathbf{0}} \cdot \mathbf{V}_{g}-\delta\right| \sim \Omega_{R}, \Omega_{B}$. Since the growth rates of SR and SB scattering instabilities, given by Eqs. (19) and (20), respectively, are proportional to $\Omega_{R}$ and $\Omega_{B}$, one notices that quantum and strong ion correlation effects significantly affect the $e$-folding time of the instabilities. Furthermore, the growth rates, which are proportional to $F_{p, l}$, are minimum at the center of the vortex pump wave with OAM.

Next, for the modulational instabilities, we have $D_{ \pm} \neq 0$ and $S_{R, B} \neq 0$. Here, we have to retain both upper and lower CPEM sidebands on an equal footing in (12) and (13), and write them as

$$
S_{R}\left[\left(\Omega-\mathbf{K} \cdot \mathbf{V}_{g}\right)^{2}-\delta^{2}\right]=\frac{\delta \omega_{p}^{2} e^{2} K^{2}\left|\mathbf{A}_{0} F_{p}(p, l)\right|^{2}}{2 \omega_{0} m_{0}^{2} c^{2}}
$$

and

$$
S_{B}\left[\left(\Omega-\mathbf{K} \cdot \mathbf{V}_{g}\right)^{2}-\delta^{2}\right]=\frac{\delta \omega_{p}^{2} Z_{i}^{2} e^{2} K^{2}\left|\mathbf{A}_{0} F_{p}(p, l)\right|^{2}}{2 \omega_{0} m_{0} m_{i} c^{2}} .
$$

Equations (21) and (22) can be analyzed numerically to obtain the growth rates of the modulational instabilities. However, some analytical results follow for $\mathbf{K} \cdot \mathbf{V}_{g}=0$, in which case we have from (21) and (22), respectively,

$$
\begin{aligned}
\Omega^{2}= & \frac{1}{2}\left(\Omega_{L}^{2}+\delta^{2}\right) \\
& \pm \frac{1}{2}\left[\left(\Omega_{L}^{2}-\delta^{2}\right)^{2}+\frac{2 \delta \omega_{p}^{2} e^{2} K^{2}\left|\mathbf{A}_{0} F_{p}(p, l)\right|^{2}}{\omega_{0} m_{0}^{2} c^{2}}\right]^{1 / 2}
\end{aligned}
$$

and

$$
\begin{aligned}
\Omega^{2}= & \frac{1}{2}\left(\Omega_{r, I}^{2}+\delta^{2}\right) \\
& \pm \frac{1}{2}\left[\left(\Omega_{r, I}^{2}-\delta^{2}\right)^{2}+\frac{2 \delta \omega_{p}^{2} Z_{i}^{2} e^{2} K^{2}\left|\mathbf{A}_{0} F_{p}(p, l)\right|^{2}}{\omega_{0} m_{0} m_{i} c^{2}}\right]^{1 / 2},
\end{aligned}
$$

which exhibit oscillatory modulational instabilities.

\section{SUMMARY AND CONCLUSIONS}

In summary, we have considered nonlinear interactions of large amplitude CPEM waves carrying OAM with electron and ion plasma modes in an unmagnetized quantum plasma, accounting for quantum forces that act on a degenerate electron fluid. On the time scale of the electron plasma period, the ions do not respond to electrostatic electron plasma waves that are driven by the CPEM wave pressure. It is shown that the electron plasma wave spectrum is significantly modified by the inclusion of the quantum statistical electron pressure, perturbations of quantum forces associated with the electron exchange and electron-correlation potentials due to spin effects, as well as that of the quantum Bohm potential through which electrons tunnel at nanoscales. Furthermore, inclusion of the dynamics of strongly correlated nondegenerate ions provides the possibility of low-frequency electrostatic oscillations that are supported by restoring forces coming from gradients of the quantum statistical electron pressure as well as electron-exchange and electron correlations potentials, and the quantum electron Bohm potential, while the ion mass provides the inertia to sustain the wave. The viscoelastic relaxation of ion correlations and ion fluid shear and bulk viscosities introduce the damping of low-frequency electrostatic ion oscillations. Both electron and ion plasma oscillations are excited by large amplitude CPEM waves due to stimulated Raman and Brillouin scattering instabilities. We also have the possibility of modulational instabilities of the CPEM waves via which nonresonant electron density perturbations are created. Hence, there are enhanced electrostatic fluctuations at nanoscales in dense quantum plasmas. In conclusion, we stress that the results of the present investigation are useful for 
understanding the salient features of enhanced density fluctuations and inhomogeneities which are nonlinearily created by large amplitude CPEM waves with OAM in laser created solid density compressed plasmas and in compact astrophysical objects.

\section{ACKNOWLEDGMENT}

This work was supported by the Deutsche Forschungsgemeinschaft through the Project No. SH21/3-2 of the Research Unit 1048 .
[1] P. K. Shukla and B. Eliasson, Phys. Usp. 53, 51 (2010).

[2] P. K. Shukla and B. Eliasson, Rev. Mod. Phys. 83, 885 (2011).

[3] S. V. Vladimirov and Yu. O. Tyshetskiy, Phys. Usp. 54, 1243 (2011).

[4] F. Haas, Quantum Plasmas: An Hydrodynamical Approach (Springer, New York, 2011).

[5] S. L. Shapiro and S. L. Teukolsky, Black Holes, White Dwarfs and Neutron Stars: The Physics of Compact Objects (John Wiley \& Sons, New York, 1983).

[6] A. M. Abrahams and S. L. Shapiro, Astrophys. J. 374, 652 (1991).

[7] S. Balberg and S. L. Shapiro, in The Properties of Condensed Matter in White Dwarfs and Neutron Stars, edited by M. Levy (Academic, London, 2000).

[8] D. Lai, Rev. Mod. Phys. 73, 629 (2001); A. K. Harding and D. Lai, Rep. Prog. Phys. 69, 2631 (2006).

[9] H. M. van Horn, Science 252, 384 (1991); V. E. Fortov, Phys. Usp. 52, 615 (2009).

[10] M. S. Murillo, Phys. Rev. E 81, 036403 (2010); D. A. Chapman and D. O. Gericke, Phys. Rev. Lett. 107, 165004 (2011).

[11] D. Kremp, T. Bornath, M. Bonitz, and M. Schlanges, Phys. Rev. E 60, 4725 (1999); V. M. Malkin, N. J. Fisch, and J. S. Wurtele, ibid. 75, 026404 (2007); H. Azechi et al., Laser Part. Beams 9, 193 (1991); R. Kodama et al., Nature (London) 412, 798 (2001).

[12] P. A. Markowich et al., Semiconductor Equations (Springer, Berlin, 1990).

[13] N. Crouseilles, P. A. Hervieux, and G. Manfredi, Phys. Rev. B 78, 155412 (2008).

[14] L. Segretain, Astron. Astrophys. 310, 485 (1996).

[15] B. DeMarco and D. S. Jin, Science 285, 1703 (2000).

[16] M. S. Murillo, Phys. Rev. Lett. 87, 115003 (2001).

[17] P. K. Shukla and L. Stenflo, Phys. Plasmas 13, 044505 (2006).

[18] P. K. Shukla and B. Eliasson, Phys. Rev. Lett. 96, 245001 (2006).

[19] P. K. Shukla and B. Eliasson, Phys. Rev. Lett. 99, 096401 (2007).

[20] D. Shaikh and P. K. Shukla, Phys. Rev. Lett. 99, 125002 (2007).

[21] L. Stenflo and G. Brodin, J. Plasma Phys. 21, 261 (2010).

[22] H. F. Wilhelm, Z. Phys. 241, 1 (1971).

[23] G. Manfredi, Fields Inst. Commun. 46, 263 (2005).

[24] C. L. Gardner and C. Ringhofer, Phys. Rev. E 53, 157 (1996).

[25] G. Manfredi and F. Haas, Phys. Rev. B 64, 075316 (2001).

[26] S. Chandrasekhar, An Introduction to the Study of Stellar Structure (The University of Chicago Press, Chicago, 1939).

[27] L. D. Landau and E. M. Lifshitz, Statistical Physics (Butterworth-Heinemann, Oxford, 1980).

[28] N. L. Tsintsadze and L. N. Tsintsadze, Europhys. Lett. 88, 35001 (2009).
[29] J. T. Mendonça, Phys. Plasmas 18, 062101 (2011).

[30] M. Marklund and G. Brodin, Phys. Rev. Lett. 98, 025001 (2007); G. Brodin, M. Marklund, J. Zamanian, A. Ericsson, and P. L. Mana, ibid. 101, 245002 (2008).

[31] L. Brey, J. Dempsey, N. F. Johnson, and B. I. Halperin, Phys. Rev. B 42, 1240 (1990); see also L. Hedin and B. I. Lundqvist, J. Phys. C 4, 2064 (1971).

[32] P. K. Shukla and B. Eliasson, Phys. Rev. Lett. 108, 165007 (2012).

[33] P. K. Shukla, Nat. Phys. 5, 92 (2009).

[34] S. H. Glenzer, O. L. Landen, P. Neumayer et al., Phys. Rev. Lett. 98, 065002 (2007); S. H. Glenzer and R. Redmer, Rev. Mod. Phys. 81, 1625 (2009).

[35] W. L. Kruer, The Physics of Laser Plasma Interactions (Addison-Wesley, Redwood City, CA, 1973).

[36] L. Stenflo, Phys. Scr. 14, 320 (1976).

[37] Y. T. Yan and J. M. Dawson, Phys. Rev. Lett. 57, 1599 (1986); Z. Huang and K.-J. Kim, Phys. Rev. ST Accel. Beams 10, 034801 (2007).

[38] E. Hemsing, A. Marinelli, and J. B. Rosenzweig, Phys. Rev. Lett. 106, 164803 (2011).

[39] P. K. Shukla, N. N. Rao, M. Y. Yu, and N. L. Tsintsadze, Phys. Rep. 138, 1 (1986).

[40] L. Allen, M. W. Beijersbergen, R. J. C. Spreeuw, and J. P. Woerdman, Phys. Rev. A 45, 8185 (1992).

[41] J. T. Mendonça, B. Thide, and H. Then, Phys. Rev. Lett. 102, 185005 (2009).

[42] D. Bohm and T. Staver, Phys. Rev. A 84, 836 (1951).

[43] V. M. Atrazhev and I. T. Iakubov, Phys. Plasmas 2, 2624 (1995).

[44] S. Ichimaru and S. Tanaka, Phys. Rev. Lett. 56, 2815 (1986); S. Tanaka and S. Ichimaru, Phys. Rev. A 35, 4743 (1987); S. Ichimaru, H. Iyetomi, and S. Tanaka, Phys. Rep. 149, 91 (1987); M. A. Berkovsky, Phys. Lett. A 166, 365 (1992).

[45] S. Ichimaru, Statistical Plasma Physics: Condensed Plasmas (Addison-Wesley, New York, 1994).

[46] Y. I. Frenkel, Kinetic Theory of Liquids (Clarendon, Oxford, 1946).

[47] P. K. Kaw and A. Sen, Phys. Plasmas 10, 3552 (1998).

[48] N. N. Rao, P. K. Shukla, and M. Y. Yu, Planet. Space Sci. 38, 543 (1990).

[49] P. K. Shukla, G. Brodin, M. Marklund, and L. Stenflo, Phys. Lett. A 373, 3165 (2009).

[50] M. Y. Yu, K. H. Spatschek, and P. K. Shukla, Z. Naturforsch. 29, 1736 (1974); P. K. Shukla, M. Y. Yu, and K. H. Spatschek, Phys. Fluids 18, 265 (1975); G. Murtaza and P. K. Shukla, J. Plasma Phys. 31, 423 (1984). 